\title{
El agua, los hoteles \\ Gran Turismo y la \\ ciencia de sistemas
}

DOI: 10.22403/UQROOMX/TYP05/01

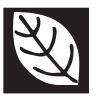

María Luisa Gómez González

Ricardo Tejeida Padilla*

\section{RESUMEN}

El fenómeno de la escasez del agua se ha agudizado en el mundo debido a la contaminación y al desperdicio. En México, dada su importancia, se le está concediendo el carácter de prioridad nacional.Todas las actividades económicas del país son usuarias constantes del agua y requieren un permanente abastecimiento del recurso para estar en condiciones de llevar a cabo sus funciones. En el sector turístico, dependiendo de su magnitud y de los servicios que ofrecen, son los hoteles los que utilizan el agua en cantidades considerables.

El presente trabajo, que forma parte de una investigación en proceso, aborda esta cuestión desde la perspectiva de sistemas con el fin de proponer una solución holística que haga frente al problema del desabasto de agua y su manejo sustentable en los hoteles de la ciudad de México, tanto para la prestación de servicios, como para preservar el vital líquido y evitar la depredación del ambiente. Con la ayuda de la Soft System Methodology y el Viable System Model se diseñó un modelo sistémico de gestión del agua para los hoteles Gran Turismo de la Ciudad de México, el cual abarca las cinco funciones esenciales para la viabilidad: implementación, coordinación, control, inteligencia y política. Se pretende establecer una nueva forma de administrar los recursos, resaltando la afinidad entre sustentabilidad y rentabilidad. \begin{tabular}{r|r} 
Palabras & Agua, hoteles, modelo de sistemas viables, sistemas, turismo \\
Clave &
\end{tabular}

*Instituto Politécnico Nacional, Escuela Superior de Turismo / marialuisa I205@yahoo.com.mx / texpa80@yahoo.com.mx 


\section{Introducción y antecedentes}

El problema del agua se vislumbra como el "mayor conflicto geopolítico del siglo XXI" ya que se calcula que para el año 2025 la demanda de suministro se elevará $56 \%$ (Frers, s.f.). Es importante señalar que, de acuerdo con la Organización Mundial de la Salud, 2.2 millones de personas mueren cada año debido a enfermedades causadas por agua contaminada y saneamiento deficiente.

La Comisión Nacional del Agua (Conagua) señala que los programas hídricos propuestos hasta ahora constituyen proyectos de inversión que representan un costo y un efecto menores a los del agotamiento del agua; además, reconoce que el agua es un recurso que debe protegerse de manera integral, lo que significa que la sociedad, las instituciones, las dependencias y los gobiernos tienen un papel que cumplir en su cuidado, por lo que destaca la necesidad de imponer el cuidado del agua como una alternativa óptima para la sustentabilidad y el beneficio común de los habitantes (Conagua, s.f.). El problema no es la falta de agua dulce potable, sino la mala gestión y distribución de los recursos hídricos y sus métodos (Frers, s.f.).

\section{Inversión y tecnología}

La disponibilidad en cantidad y calidad, el uso y el manejo del agua, son áreas de la ciencia y la tecnología. Entonces, es preciso fortalecer los recursos humanos y materiales con los que se cuenta y desarrollar nuevas capacidades. La utilización de estos recursos requiere además un cambio en la perspectiva sobre la investigación, pues, ante un desafío como el que representa el agua, es necesario pasar de los esfuerzos individuales y monotemáticos al desarrollo de grandes proyectos multidisciplinarios con una visión de largo plazo y que puedan ser aplicados de manera eficaz en tres grandes campos: a) la disponibilidad, b) el uso racional y c) la gestión (Flores, 2006: 200-20I).

La gestión del agua en la Ciudad de México es un proceso que tiene sustento en un conjunto de principios, derechos, bienes y responsabilidades que el Estado, las organizaciones y los usuarios manejan de forma coordinada para alcanzar el desarrollo sustentable, es decir, la preservación del equilibrio hidrológico, el aprovechamiento y la protección de los recursos hídricos, de 
modo que no se comprometa la satisfacción de los requerimientos de agua de las generaciones futuras.'

La gestión del agua tiene repercusiones ambientales, económicas, culturales, fiscales y urbanas. La subregión Valle de México registra una sobreexplotación anual de los acuíferos de $979 \mathrm{hm}^{3}$ (Conagua, 2003).

Los hoteles no están exentos del problema, pues utilizan una gran cantidad del líquido -alrededor de 881000 litros diarios en una red de infraestructura que consta de 44509 habitaciones en 589 hoteles (Delgado, 2005)-. Marlene Ehrenberg Enríquez, directora del Programa ConservAgua del Colegio Mexicano de Ecoturismo, destaca que mientras una persona en la Ciudad de México consume en promedio 200 litros de agua al día, los hoteleros consumen I 000, cifra alarmante si se considera que este sector crece más de $12 \%$ al año. No obstante, siguen siendo pocos los hoteles que se certifican en materia ambiental y que observan un manejo sustentable del recurso, ya que la certificación es voluntaria (Sectur, s.f.).

Por otra parte, el Programa Agenda 21 para el Turismo Mexicano surge con la intención de buscar la sustentabilidad en la prestación de los servicios turísticos, y en uno de sus apartados aborda la necesidad de que los hoteles fortalezcan sus procesos de uso y aprovechamiento de agua como una posible solución; sin embargo, esto sólo ocurre en el nivel de promoción por parte de la Secretaría de Turismo (Sectur).Además, el programa de conservación de agua en hoteles sigue siendo voluntario (Sectur, s.f.).

El programa permite la certificación en calidad ambiental turística, y el organismo que la otorga es la Procuraduría Federal de Protección al Ambiente (Profepa), la cual emite una guía de autoevaluación ambiental con la finalidad de que los interesados tengan conocimiento de las obligaciones legales en esta materia. Empero, solamente algunas empresas de hospedaje cuentan con plantas de agua o de tratamiento en sus instalaciones, buscando dar un manejo sustentable y no depredatorio al recurso. Esto es, aunque es urgente contar con una adecuada gestión del agua, la mayoría de los hoteles no actúan en consecuencia y la aplicación de esta medida continúa supeditada a su voluntad. Adicionalmente hay que apuntar que no se han otorgado los apoyos ni los estímulos necesarios para tal gestión en el sector turístico.

'Diario Oficial de la Federación, 29 de abril de 2004. 


\section{Metodología}

El método utilizado en esta investigación es el de sistemas, a través de la Soft System Methodology (Checkland y Scholes, 1990; Checkland, 1993), integrando fases cibernéticas de entrada, proceso y salida. Se hizo una interfaz, en la parte de modelos conceptuales, con elViable System Model (Beer, 1994), compuesto por tres estados que engloban la gestión en el ambiente, el área de operación y el metasistema. Este modelo se utilizó porque se trata de un sistema social organizado con demandas de homeostasis en un ambiente entrópico.

La Soft System Methodology (SSM) ayudó a conformar la definición básica de los sistemas relevantes, emanados de la visión enriquecida del problema del agua en los hoteles Gran Turismo de la Ciudad de México. Una vez encontrados los sistemas relevantes, se utilizó el Viable System Model (VSM) con el fin de llegar a un modelo homeostático.

\section{Resultados}

La aplicación de la SSM y del VSM permitió conformar un modelo integrador nombrado Modelo de Gestión del Agua para los Hoteles Gran Turismo de la Ciudad de México, el cual, de manera resumida, se describe a continuación:

- Sistema I. Está representado por el uso y aprovechamiento del agua; es el sistema que está en intercambio de información con el entorno actual. Los niveles del hotel involucrados en el uso y aprovechamiento del recurso son el técnico, el operativo, el administrativo y el gerencial, los cuales son señalados como parte de las actividades de aseo y mantenimiento, manejo higiénico de alimentos y la imagen corporativa, es decir, se incluyen todos los procesos y usos del agua. El agua es empleada a todos los niveles: el cliente y los niveles técnico, operativo y gerencial. Este último repercute en una imagen corporativa del hotel vinculada con todos los niveles, señalando el manejo sustentable del agua, el ahorro y, en general, la cultura y la administración del recurso.

- Sistema 2. Constituye la coordinación entre las actividades del sistema I y las del 3.

- Sistema 3. Referente a la estrategia financiera que abarca la contabilidad y el manejo de finanzas del hotel, esencialmente la asignación de recursos 
y la rendición de cuentas. En este caso, incluye el abatimiento de costos y el aprovechamiento de recursos, vinculando la rentabilidad con la sustentabilidad. La auditoría es la encargada de la revisión. En este sistema se encuentran las obligaciones fiscales -que debendeclararse al Sistema de Administración Tributaria (SAT) - y los trámites ante la Sectur: el registro al que deben estar inscritas las empresas de hospedaje (Registro Nacional de Turismo, RNT) y, en lo que respecta a la Certificación en Calidad Ambiental Turística, las certificaciones que se promueven a través de la Agenda 21 de conformidad con la Profepa. Se haría hincapié en la necesidad de la intervención del Sistema de Aguas Nacionales en este rubro.

En lo que respecta a la Certificación en Calidad Ambiental, la Profepa es la autoridad competente para realizar la auditoría en materia ambiental. En cuanto a la auditoría financiera, operativa y administrativa, es la propia empresa la que designa un auditor interno para llevarla a cabo. En los casos que el SAT juzgue pertinentes, se efectúa una auditoría externa de acuerdo con los supuestos enunciados en la ley (número de empleados, nivel de ingresos, etcétera).

- Sistema 4. Es la administración de la tecnología; representa la inteligencia y la innovación tecnológica que tiene que adaptarse a las condiciones impuestas por el medioambiente, con la finalidad de lograr un desarrollo tecnológico, es decir, las ecotecnias para el desarrollo turístico sustentable; este sistema es el que mantiene el intercambio de información con el entorno futuro.Aquí se señala la necesidad de contar con plantas potabilizadoras y de tratamiento de agua, entre muchas otras tecnologías.

- Sistema 5. Es imprescindible un consejo de administración multidisciplinario que trate lo concerniente a la visión, la identidad y las políticas de contingencia adoptadas por la empresa, a fin de estar en condiciones de afrontar circunstancias y situaciones del entorno (de tipo laboral y ambiental); un presupuesto para siniestros considerado en la contabilidad de la empresa, por medio de la creación de reservas de contingencia -por ejemplo las propuestas por el gobierno mediante el Fondo Interamericano de Asistencia para Situaciones de Emergencia (Fondem).

El modelo propuesto presenta las cinco funciones esenciales para la viabilidad; en la figura I se reproduce el pictograma. 
El agua, los hoteles Gran

Turismo y la ciencia de sistemas

14

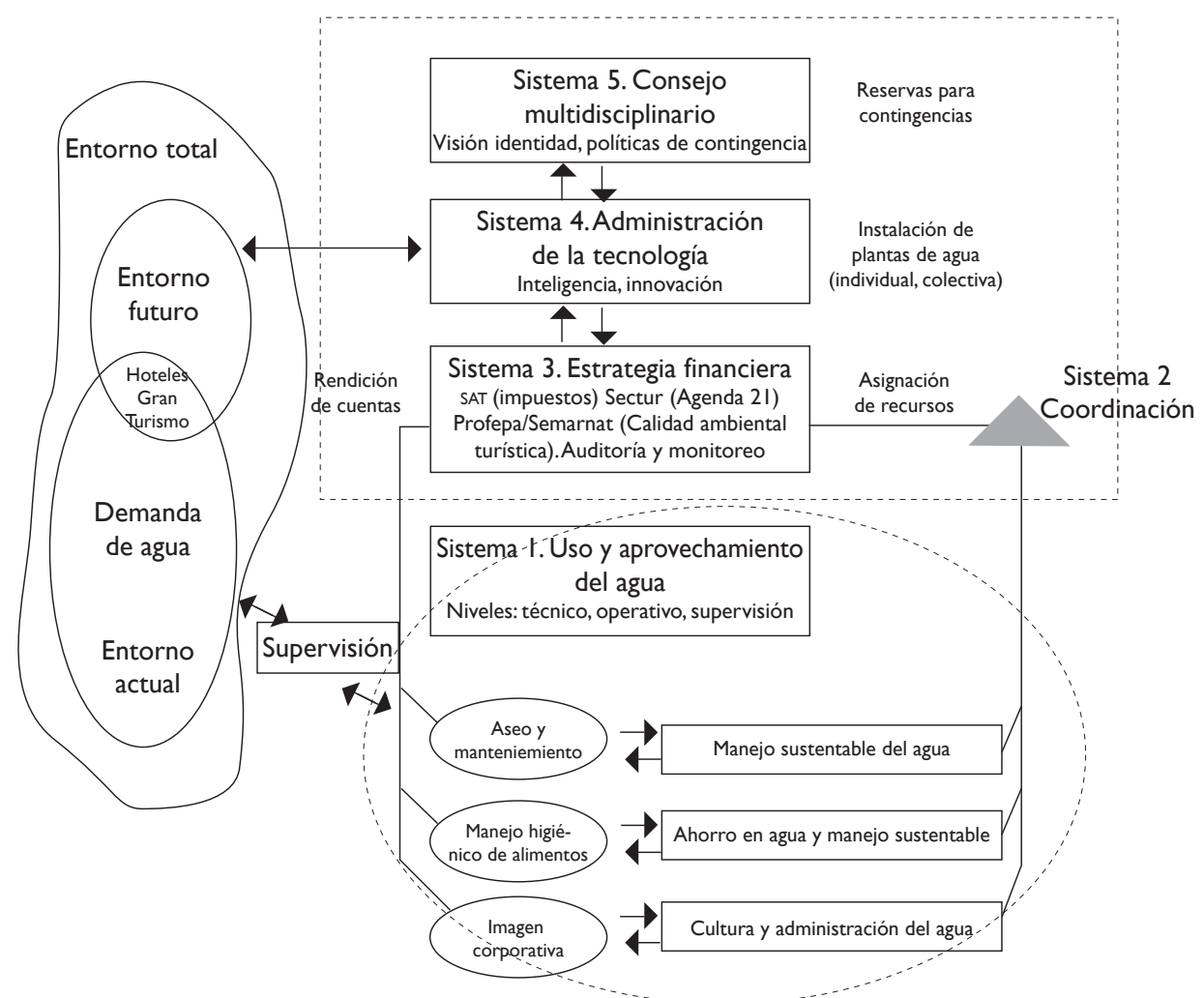

Fuente: Elaboración propia a partir de la SSM y el vSM.

Figura I. Modelo de gestión del agua para los hoteles Gran Turismo de la Ciudad de México

\section{Discusión}

Aun cuando la gestión del agua en la Ciudad de México se concibe como un conjunto de reglas que mediante un proceso pretende alcanzar el desarrollo sustentable y preservar el equilibrio ecológico buscando satisfacer las necesidades de futuras generaciones, la realidad es otra. De los diversos problemas, destacan la 
reducción de áreas de recarga, la sobreexplotación y hundimientos diferenciales, la importación de cuencas cercanas, escasos volúmenes de agua tratada, entre otros. Aunado a lo anterior (causas hidrofísicas) se encuentran los problemas relativos a la sociedad (causas sociopolíticas), como el crecimiento acelerado de población con una inadecuada cultura del cuidado del agua y los consecuentes usos inapropiados del vital líquido, lo que ocasiona la sobreexplotación de su acuífero ante por la continua demanda para el consumo.

\section{Conclusiones}

Debe subrayarse que, ya sea desde el punto de vista económico, social, ecológico o turístico, el agua es un asunto sobre el cual es necesario adoptar medidas eficaces e inmediatas, sobre todo porque el suministro de agua es un "servicio" que no tiene sustituto.

Aun cuando el problema del agua requiere acciones multidisciplinarias, la actuación ha sido más intensa por parte de la ingeniería, los ambientalistas y los economistas; la participación del sector turístico aún es marginal, debido al carácter voluntario de la adopción de medidas de manejo sustentable del agua. En concreto, son menos de diez los hoteles que están registrados en el Programa de Calidad Ambiental Turística en la Ciudad de México (según datos de la Sectur en el 2006), cifra que es mínima considerando que los hoteles utilizan gran cantidad de agua (aproximadamente 88I 000 litros de agua diarios en una red de infraestructura de 44509 habitaciones en 589 hoteles).

Por otra parte, la Sectur, en un intento por alcanzar el desarrollo turístico sustentable, emite la Agenda 2 I con su programa de conservación de agua en establecimientos de hospedaje y otros servicios turísticos y el de certificación para hoteles; sin embargo, no hace hincapié en la necesidad de instalar plantas de agua para el abastecimiento del recurso y en el beneficio que representa para la comunidad.

La certificación sigue siendo optativa y cumplir sus parámetros no es fácil ni se cuenta con un antecedente o una guía para la certificación, como las normas iso o algún otro sistema de calidad.

Por lo anterior, existe la permanente necesidad de abastecimiento de agua, y surge el problema de la escasez por diversas causas, entre las que resalta la falta de plantas potabilizadoras y de tratamiento.Asimismo, la Conagua no define 
los usos del agua en los hoteles, ni tampoco hay un programa de apoyo para la instalación de plantas en los hoteles de la Ciudad de México ni un financiamiento o subsidio para lograr un desarrollo sustentable. En resumen, la clave está en la investigación, la inversión y la tecnología: una solución multidisciplinaria.

\section{FUENTES CONSULTADAS}

Beer, S. (1994). Beyond Dispute:The Invention ofTeam Syntegrity. Chichester:John Wiley.

Checkland, P. (1993). Pensamiento de sistemas, práctica de sistemas. México: Limusa.

— y J. Scholes (1990). Soft System Methodology in Action. Chichester:John Wiley.

Comisión Nacional del Agua (Conagua) (s.f.) “La gestión del agua en México: avances y retos" [en línea]. Disponible en: http://www.cna.gob.mx/ eCNA/Espaniol/Directorio/Default.aspx [2007, 20 de febrero].

- (2003). Estadísticas del agua en México 2003. México:Sistema Unificado de Información Básica del Agua, Comisión Nacional del Agua.

Delgado, M. (2005). "Comisión Especial para la Gestión Integral del Agua" [en línea]. Foro Turismo y Agua en el Distrito Federal, celebrado el 3 de noviembre de 2005. México. Disponible en: http:www.martha.org. $\mathrm{mx} /$ eventos/eventos/forotusimoagua.html [2006, 3I de octubre].

Diario Oficial de la Federación (2004). 29 de abril de 2004.

Flores, J. (2006). "Requiere esfuerzo científico la preservación de nuestros recursos: debe la sociedad reclamar más fondos para investigación” en Fabrizio León Diez (ed.) Agua emergencia en puerta. México: La Jornada, $335 \mathrm{p}$.

Frers, C. (s.f.). “La próxima guerra... la guerra del agua” [en línea]. Ecojoven. Disponible en:http://www.ecojoven.com/tres/ I0/acuiferos.html [2007, 13 de marzo].

Secretaría de Turismo (Sectur), "Agenda 21 para el Turismo Mexicano” [en línea]. Disponible en: http://www.sectur.gob.mx/wb2/sectur/sect_ 8763_agenda_2I_para_el_tu [2006, I7 de octubre]. 